\title{
Understanding Mobile Searcher Attention with Rich Ad Formats
}

\author{
Dmitry Lagun \\ Google \\ dlagun@google.com
}

\author{
Donal McMahon \\ Indeed \\ dmcmahon@indeed.com
}

\author{
Vidhya Navalpakkam \\ Google \\ vidhyan@google.com
}

\begin{abstract}
Mobile Search experiences have evolved significantly from a few blue links that require users to click. Recent search and ad units surface instant information to the user in a variety of visually rich formats that include images, horizontal swipes, and vertical scrolls. These innovative experiences call for new metrics and models to better understand searcher behavior on mobile phones. In this paper, we study how the presence of ads and their formats impacts searcher's gaze and satisfaction. We systematically vary presentation format of the sponsored result, while controlling for other factors, such as position and quality of organic results. We experiment with several configurations of text ad and rich ad formats. Our findings indicate that showing rich ad formats improve search experience, by drawing more attention to the information-rich ad and allowing users to interact to view more offers, which increases user satisfaction with search. In addition, we extend prior work by comparing the performance of various models to infer user's gaze from viewport data. Our models improve accuracy of existing viewport-based gaze inference methods by $30 \%$ in Pearson's correlation. Together, our findings show that viewport data can be used for fast, accurate and scalable measurement of user attention on a per-element basis, for both ads as well as organic search results.
\end{abstract}

\section{Keywords}

user attention; eye tracking; viewport; ads; user study

\section{INTRODUCTION}

Given the rapid growth of mobile Search traffic, coupled with recent innovations towards surfacing instant information to the user in a variety of visually attractive and rich UIs, it is clear that Search and Advertising have come a long way since the days of the few blue links. Consider a specific example such as comparative shopping. Earlier, users had to click through multiple ads for comparative shopping. Today, there's a convenient answer-like rich ad unit that sur-

Permission to make digital or hard copies of part or all of this work for personal or classroom use is granted without fee provided that copies are not made or distributed for profit or commercial advantage and that copies bear this notice and the full citation on the first page. Copyrights for third-party components of this work must be honored For all other uses, contact the owner/author(s).

CIKM'16 October 24-28, 2016, Indianapolis, IN, USA

(C) 2016 Copyright held by the owner/author(s).

ACM ISBN 978-1-4503-4073-1/16/10 . .\$15.00

DOI: http://dx.doi.org/10.1145/2983323.2983853

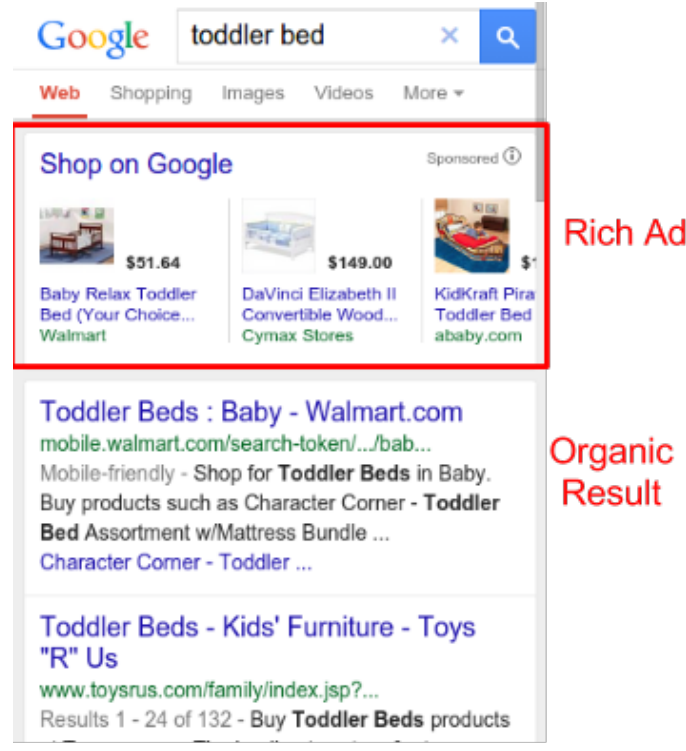

Figure 1: An example of rich format for the shopping Ad.

faces all the relevant information including product name, image, price and merchant information. Moreover, it efficiently packs such information for multiple offers into an interactive UI, such as shown in Figure 1. Users can swipe such units to view more offers in the same vertical space, which is a revolutionary shopping experience compared to the traditional experience of a few blue links.

How do we evaluate such rich, answer-like experiences? What is their effect on users' gaze? More importantly, how do they impact search satisfaction for queries with commercial intent? Answering these questions is critical for understanding mobile searcher behavior, which forms the basis for developing better metrics and models of user satisfaction with interactive UI that go beyond click interactions.

Measuring users' eye gaze on search results page (SERP) can offer useful insights into user behavior, as has been demonstrated in several previous studies on desktop [4, $24,6,14,21,8,23]$. For example, these studies showed that users' attention decays with result position as user examines the SERP. It is unclear whether these insights, obtained in desktop setting, hold for mobile phones, especially in the presence of ads and their increasingly diverse formats. There are several possibilities. If users tend to skip ads, the 
ad unit may draw less attention compared to the top organic result. On the other hand, if users always scan in a top-down fashion, we may not see any difference in attention across positions in the presence vs. absence of ads. A third possibility is that if users find the rich format ad more relevant, the ad unit might draw more attention than regular text ads or organic results. Each of these has different implications for understanding searcher attention in presence of ads and determining ad's relevance to the user.

Moreover, studies of searcher attention has been fertile ground for research. Several works have demonstrated its utility for predicting search result relevance [17, 12] and quality of search result snippets [19]. Searcher attention has been very informative for evaluating quality of instant answers [22] and rich information panels (e.g., based on knowledge graph result) [25]. Measuring mobile searcher attention at scale remains extremely challenging: commercial eye-tracker systems are expensive and in-lab studies are time-consuming. As such, previous work explored other scalable methods that could approximate user attention at scale. In desktop settings, many researchers (e.g., [16, 25, 21]) used mouse cursor to approximate user attention. However, lack of mouse cursor on mobile devices makes attention measurement at scale more challenging. Some researchers (e.g., [15, 22]) suggested to use viewport, i.e., the portion of the web page that is visible on the phone screen at a given point in time, to approximate user attention on search results. We build upon previous work by developing better models to accurately infer users' gaze from viewport data.

This paper makes the following contributions:

- presents the first quantitative study to measure users' gaze and satisfaction with answer-like swipeable ads in mobile phones

- shows that rich format ads receive significantly more attention compared to regular text ads or organic results at the same position, and better satisfy users compared to showing no ads.

- presents multiple models to accurately infer users' gaze on a per-element basis from viewport data

Thus we show that viewport data can be used to perform fast and scalable measurement of user attention on search results. We begin by surveying related work in eye tracking and user behavior for search on desktops and mobile devices. We then describe our experiment and user study, followed by the analysis of the user study data. We conclude with a discussion reviewing the findings and limitations of this study, along with suggestions for future work.

\section{RELATED WORK}

Our related work spans two lines of research: eye tracking in search examination studies and gaze inference models.

Eye tracking technology has been extensively used in studies of web search result examination behavior in desktop settings. Granka et al. [9] studied how users browse search results and select links. They showed that users spend most of the time inspecting the first and the second result before their first click. Lorigo et al. [24] used eye tracking to study gaze trajectories on a search results page in more detail. They found that only $25 \%$ of users examine search results in the order they are presented by the search engine.
A similar study was conducted by Guan and Cutrell [10], who showed the effect of target result position on searcher's examination behavior. Recently, eye tracking method was used to study user interactions with automatic query completion interface [14] and word acquisition process in query reformulation [8].

Despite the abundance of research about searcher's attention on desktops, attention on mobile devices remained relatively unexplored. Huang and Diriye [15] discussed the potential utility of viewport logging on touch-enabled mobile devices. In this paper, we use client based viewport logging similar to [15] to track user interactions on the search result page. Biedert et al.[3] is one of the first quantitative eye tracking study of reading behavior performed on an actual mobile device. They reported that user attention is distributed almost uniformly over the the mobile phone viewport. More recently, Lagun et al. [22] conducted an eye tracking of search result examination of Knowledge Graph results on a mobile phone. According to them, user attention is distributed unequally during search tasks, with significant portion of attention spent in top $2 / 3$ of the mobile screen.

Apart from organic search results, previous work explored user attention and search behavior and their relation to ads and rich informational panels in the desktop settings. In a controlled study Buscher et al.[4] investigated the effect of ad quality on searcher's receptiveness to the advertisements. They found that when ad quality varied randomly, users paid very little attention to the ads. Navalpakkam et al. [25] conducted a controlled study where they varied the presence and relevance of a rich informational panel placed to the right of organic search results. They found that the information panels containing information relevant to the user's task attract more attention and longer mouse cursor hovers. Similar findings were reported by other researchers $[7,23]$. Lagun et al. [22] conducted a study on Knowledge Graph results in mobile setting, effectively replicating previous finding of [25] by replacing cursor hover time with amount of time result was visible to the user. In this paper we extend the prior work and study user attention during search tasks with answer-like sponsored results that enable horizontal swipe interactions.

Another line of research focuses on overcoming inherit limitation of costly and laborious eye tracking studies, and measuring user attention at large scale. In particular, mouse cursor tracking emerged as a cheap alternative to eye tracking by inferring searcher gaze position from mouse cursor position on desktop. The relationship between cursor and gaze has been studied empirically [5, 26, 11, 16, 25]. More recently, authors of [21] further extended this line of research and incorporated web page content into gaze prediction model. One of the reasons of why cursor tracking was so successful on desktop, is that gaze and cursor appear to be well coordinated during user actions, e.g., link clicking or text selection, as reported by Chen et al. [5]. However, when the cursor remained inactive, the reported accuracy of attention measurement degraded. While cursor tracking methods are quite successful on desktop, they are inapplicable for gaze inference on mobile interfaces, since mouse cursor is virtually non existent on mobile devices. Our previous work [22] extended this line of research to mobile device setting, where they used the amount of time a particular result was visible to the user, calling it viewport time, as a proxy of user attention, and reported relatively 
strong correlation with view time on result measured with eye tracking. More importantly, they explored several ways to attribute viewport time to a particular result (since several results may appear in the same viewport), noting that the highest correlation is achieved when viewport time on result is weighted using fraction of screen area occupied by the result. In this paper we build upon the prior work and significantly improve accuracy of gaze inference methods on a mobile interface. In addition, we explore different slice of search intents, focusing more on shopping queries, and demonstrate that gaze inference methods work reasonably well on a interactive swipe-able interfaces.

\section{USER STUDY}

In order to evaluate our hypotheses about how user attention may change in presence of rich ad formats, we designed and conducted a user study in the lab to collect data for analysis. While we focused on commercial queries with shopping intent, we tried to diversify the range of user tasks from broad, research-oriented tasks, such as "Which shoe cabinet would you purchase?" to specific tasks asking to find price information about a particular product such as "What is the lowest price you can quickly find for xbox one?". In natural setting, outside of the lab, research tasks may require substantial time to complete, thus, limiting number of participants we are able to recruit for the study. Therefore, to make the data collection feasible, we limited overall task duration to two minutes (for both types of tasks) and ensured that each task has at least several highly relevant results, navigating to which users may locate product of interest. This amount of time allowed the study participants to find and compare 2-3 products of interest during the task.

In the study we used the following protocol. Participants were first presented with a web page displaying a description of their tasks. On the same page we provided two hyperlinks - one pointing to the search results page (with a predefined query related to each task), and the second pointing to the post-task questionnaire. Participants were instructed to read the task description, and complete the task. After task completion, they filled out the post-task questionnaire where they provided their search satisfaction ratings on a likert scale from 1 (very dissatisfied) to 5 (very satisfied); the midpoint (3) represented "neither satisfied nor dissatisfied". Each participant performed 20 tasks.

To ensure that the tasks had similar levels of difficulty, two authors of the paper verified that for each task, the corresponding search results page (SERP) provided result of good quality allowing participants to complete the task in under two minutes. Thus, the tasks were fairly easy and participants were instructed to spend not more than two minutes per task. Upon completing the task, participants were asked to navigate back to the study home page by using the "Back" button on the phone, and follow the second hyperlink to complete the post-task questionnaire. Note that in this study, we predefined the queries for each task, and query reformulation was not allowed.

Our study used a within subject design with one factor representing the ad configuration (presence of the ad, or its format). The four experimental conditions, i.e., levels of the independent variable, were $\{$ no ads, text ad, rich ad, rich ad + text ad\} (i.e., a rich ad appearing above a regular text ad). We used block randomized design with five tasks per condition. The task presentation order was randomized to

\begin{tabular}{|c|c|}
\hline \#Users & 24 \\
\hline \#Queries & 750 \\
\hline \#Gaze Fixations & 28,639 \\
\hline \#Viewports (sampled every 200ms) & 34,605 \\
\hline
\end{tabular}

Table 1: Counts describing size of the data.

eliminate any learning or task order effects. As we varied the presentation (or absence) of the ad, the rest of the results on the page remained the same across all four conditions. In order to familiarize participants with the mobile device and the study flow, each participant completed 2 practice tasks prior to starting the study. After completing every 5 tasks, participants were offered a short break.

\subsection{Participants}

We recruited 30 participants with informed consent (17 male and 13 female) aged 18-65, with various occupations and self-reported mobile search experience. Most of the participants had normal or corrected vision (e.g. contact lenses) and were able to read from the mobile phone without wearing glasses. Data from 6 participants was excluded due to calibration problems with the eye tracker (missing fixations, poor calibration accuracy). Hence, in the rest of this study, we analyze data from the remaining 24 participants.

\subsection{Apparatus}

We used the SMI Glasses eye-tracker [1] to record participant's eye gaze movements. The eye-tracker allowed us to record eye gaze with a frequency of $60 \mathrm{~Hz}$ and accuracy of $0.5^{\circ}$ of visual angle [1]. We used Nexus 5 mobile phone running Android operating system. We used the Chrome browser to display the task description page and search result pages. Unlike previous studies that fixed the mobile phone $[3,22]$, we allowed participants to move the phone freely (as they would in natural settings, while being seated). The experiment began by calibrating eye gaze of each participant using a three point calibration on the screen of the mobile phone. To map gaze position into the phone coordinate system we used proprietary software to detect the phone's planar coordinates from the eye tracker front facing camera and translate gaze position into the screen coordinate system using perspective correction [2].

\subsection{Viewport Logging}

To record the exact information that was displayed on the phone screen at any given time, we instrumented custom viewport logging for the purposes of this lab study. This allowed us to record the portion of the web page currently visible on the screen, as well as bounding boxes of all search results shown on the page. Viewport logging was instrumented with JavaScript and inserted into every SERP shown to the users. Our script recorded bounding boxes of the search results, shortly after the page was rendered in the browser, and logged viewport change events such as scrolling and zooming. The instrumentation recorded the state of the viewport every $200 \mathrm{~ms}$. All the viewport events were buffered and subsequently sent with an HTTP request to a lab study server where they were stored for subsequent analysis. Such instrumentation allowed us to reconstruct what the user saw on the screen at any point of time. Table 1 summarizes key counts describing the size of our dataset. 


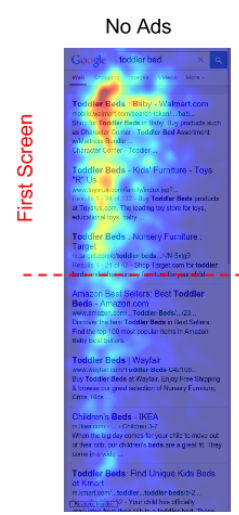

(a)

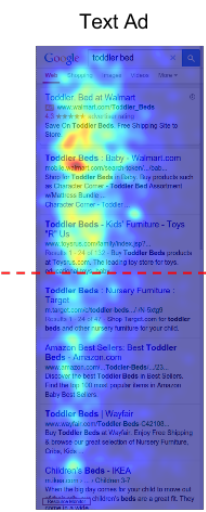

(b)

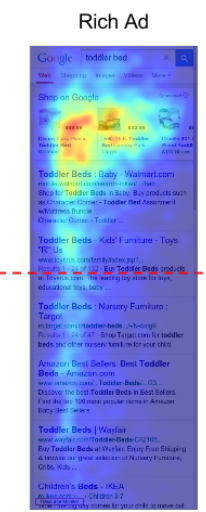

(c)

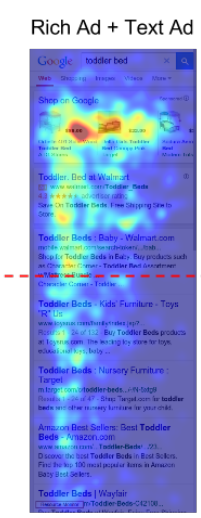

(d)
Figure 2: Heatmaps of user attention in four experimental conditions. (a) No Ads, (b) one Text Ad, (c) one Rich Ad format, (d) one Rich format co-appearing with one Text Ad.

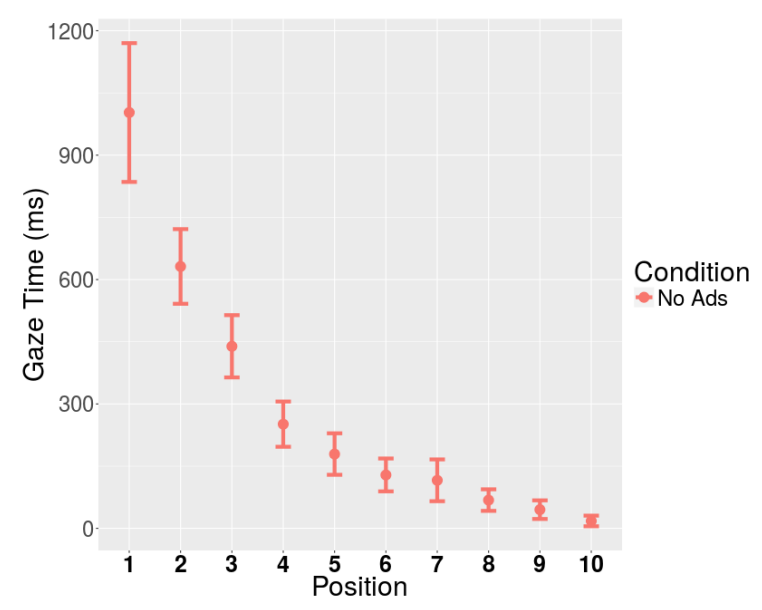

Figure 3: Distribution of gaze time over result positions for No-Ads condition.

\section{RESULTS}

In this section we focus on answering the main questions in the user study on how ad format impacts gaze and user satisfaction with search.

\subsection{Effect of Ad Format on Gaze}

We start by analyzing the gaze data aggregated over all users. Figure 2 presents a heatmap visualization of gaze distribution across the search results page, for the four experimental conditions in the study. Locations of high gaze density are shown in red while low gaze density is shown in blue. For easier visualization, we superimposed each heatmap on a screenshot of a sample search results page from the corresponding experimental condition. It is worth noting, that the heatmap shows gaze data aggregated across all queries - not for the query shown in the page screenshot. The heatmaps clearly show that there is substantial variation of attention among the four conditions, with large portion of user attention being spent on rich ad (when it appears). However, it's hard to establish statistically significant differences in attention based on the heatmaps alone,

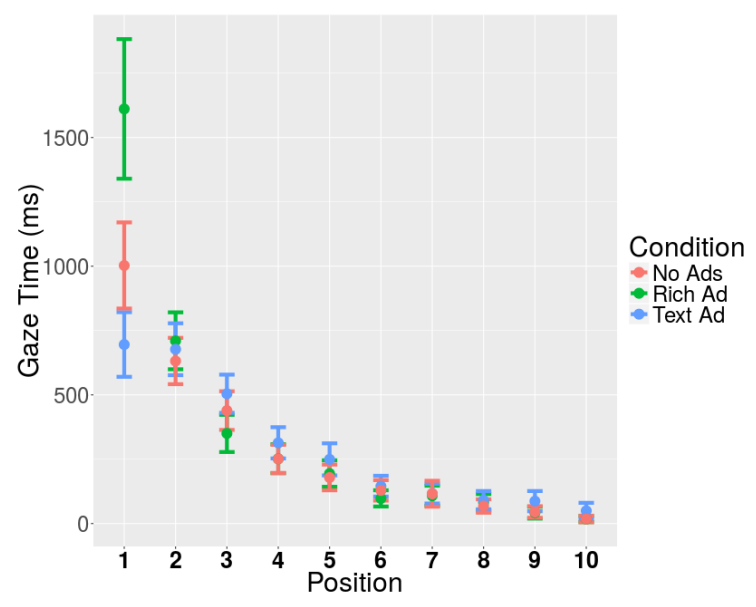

Figure 4: Distribution of gaze time over result positions for No-Ads, Rich Ad and Text Ad conditions.

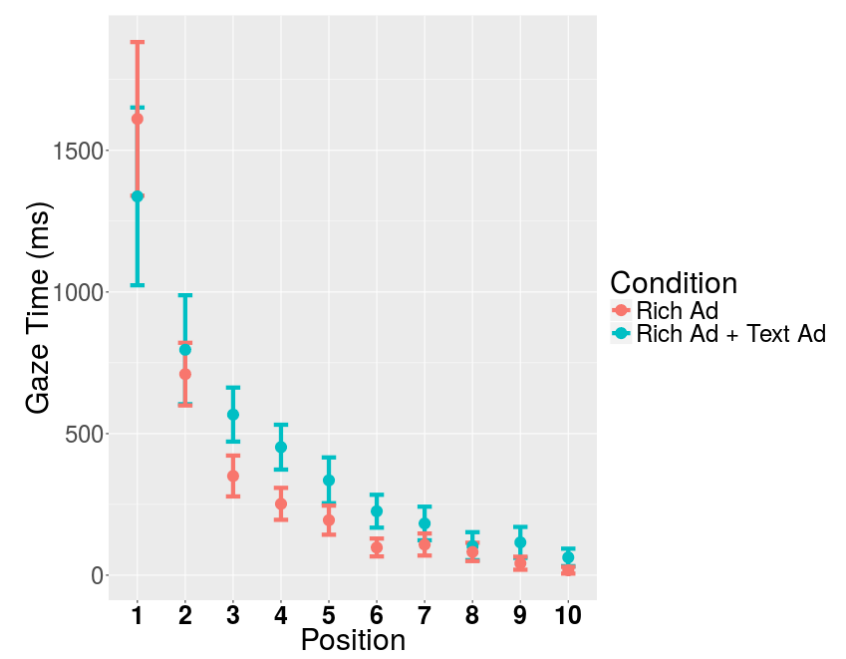

Figure 5: Distribution of gaze time over result positions for Rich Ad and combined Rich Ad + Text Ad conditions.

therefore we proceed with statistical analysis of gaze spent at each rank position.

As shown in Figure 3, we found a strong effect of position on attention $(\mathrm{p}<0.001 ; \mathrm{F}(9,2220)=75.92)$. This shows that on mobile phones, in the absence of an ad, attention decays with position, similar to findings in desktop settings [24]. In particular, the top 2-3 results get significantly more attention compared to bottom results: position 1 vs $4(\mathrm{p}<0.001$, $\mathrm{t}=72.8)$, position 2 vs $5(\mathrm{p}<0.001, \mathrm{t}=77.1)$ and position $3 \mathrm{vs}$ $6(\mathrm{p}<0.001, \mathrm{t}=53.4)$. This is not surprising since the results at top positions are expected to be of higher quality and relevance than results at lower positions.

Fig 4 shows the distribution of attention across position for the no ads condition (in red) vs. text ad conditions (in blue). We found that the top text ad gets significantly less attention than a web result at the same position $(\mathrm{p}=0.015$; $\mathrm{F}(1,226)=5.985)$. In addition, there is no noticeable difference between gaze time on text ad and the adjacent web result (i.e., position 1 vs 2 ), whereas such difference is clearly 


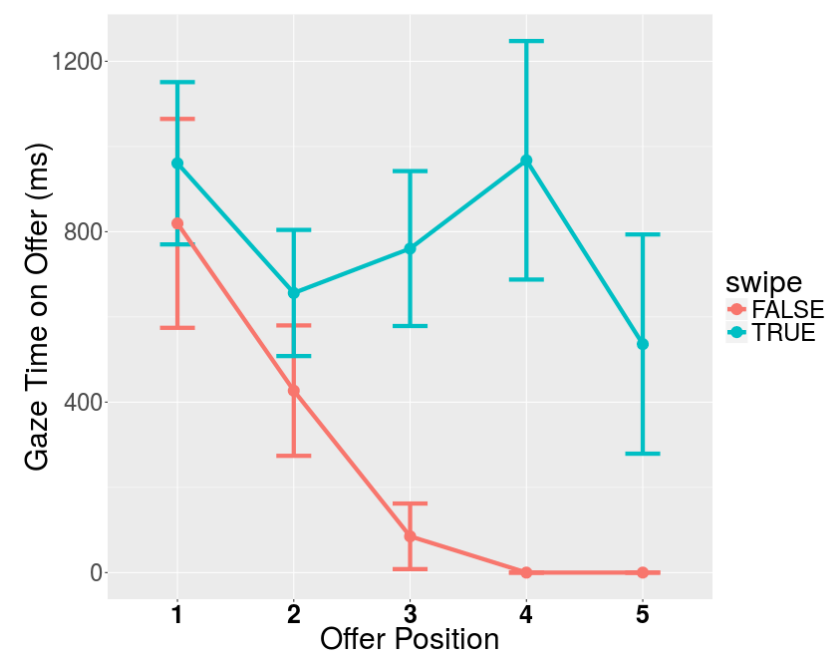

Figure 6: Average gaze time on each offer inside of Rich Ad.

present in no ads and rich ad conditions. To our knowledge, this is the first confirmation of this finding on mobile phones. We discuss possible reasons for this in the discussion section.

In contrast to the above, as shown in Fig 4, we found that a rich format ad on the top position (shown in green) gets significantly more attention than a web result at the same position (shown in red) $(\mathrm{p}=0.007 ; \mathrm{F}(1,228)=7.243)$. There's no significant difference in attention at other positions.

A natural question is how does the text ad condition compare against the rich format ad condition. Fig 4 shows that the rich format ad gets significantly more attention than the text ad at the same position $(\mathrm{p}<0.001 ; \mathrm{F}(1,226)=24.14)$. Attention on other positions remains relatively unchanged the error bars overlap at many positions.

How do the above results change if we increase the prominence of the ad block as a whole, by simultaneously presenting the rich format ad with text ad at the top positions? As shown in Fig 5, we found that attention on the rich ad (for a fixed position) was slightly lower when it was co-presented with the text ad (although not a significant difference, $\mathrm{p}=0.719 ; \mathrm{F}(1,190)=0.13)$. This may be partly due to an increased tendency for users to skip the ad block, given its higher prominence. Note, however, that we cannot directly compare the attention on the web results between these conditions (since the web results are shifted down by one position in the rich ad + text ad condition, compared to the rich ad only condition, thus differences may be confounded by result quality).

\subsection{Effect of horizontal position on Gaze}

Unlike other studies, which mostly focused on distribution of attention along vertical dimension of the SERP, we analyzed how attention is distributed horizontally inside the rich ad. The rich ad consists of multiple sub-results or offers, shown along the horizontal dimension. Upon page load, two offers are fully visible and the third is only partially visible. The user can swipe the carousel to view more offers. Figure 6 shows the average gaze time for each offer position inside the rich ad (error bars show the standard error of the mean). We show two curves, depending on whether the rich ad was

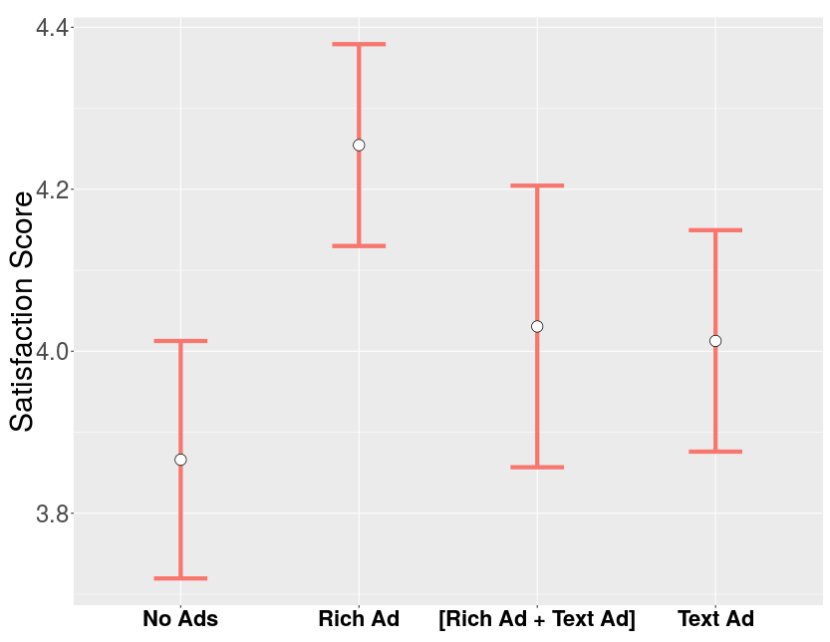

Figure 7: Average satisfaction ratings grouped by experimental condition.

swiped or not. In the absence of a swipe, we found that attention decays rapidly along the horizontal dimension. The extremely small time on the third offer can be explained by its partial visibility - upon page load only half of it is visible to the user (see Figure 1). In contrast to the sharp decay in attention in the absence of swipe, we found that the attention distribution is completely different when the rich ad was swiped. We found that there was no steep decay in gaze time per offer along the horizontal dimension, instead, there was no statistical difference across gaze time at the different offer positions. As search engines continue to improve their result presentation formats, this observation can be quite useful for inferring which offers inside a rich ad unit were viewed by the user.

\subsection{Effect of Ad Format on Satisfaction}

We posed the following hypotheses for how ads presentation may impact user satisfaction:

- H0: for commercial queries, showing any high-quality ad (regardless of format) improves user satisfaction.

- H1: for commercial queries, richer ad formats improve user satisfaction compared to traditional text ad formats.

- H2: for commercial queries, showing two ads is better than showing one ad with respect to user satisfaction.

To test H0, we compared the satisfaction scores in the no ad condition to the data combined from text ad and rich ad conditions. Overall, we found that user satisfaction responses differed significantly across conditions (ANOVA, $\mathrm{p}=0.0105$ ). Using a post-hoc Tukey HSD test, we found that showing a rich ad significantly improved user satisfaction compared to showing no ad $(\mathrm{p}<0.005)$. In contrast, showing a text ad did not significantly improve user satisfaction compared to showing no ad $(\mathrm{p}=0.74)$.

The above finding that rich ads satisfy users better than no ads, prompts us to test whether rich ads satisfy users better than traditional text ads (i.e., hypothesis H1). Although 
the trend does support H1, we did not find a significant difference in user satisfaction between showing a rich ad format vs. text ad format $(\mathrm{p}=0.068)$.

Given commercial intent of the queries and high relevant of the ads (the sponsored results are not less valuable than organic results), we tested whether showing two ads may satisfy users better than showing one, i.e., hypothesis H2. To this end, we compared the data when rich ad co-appeared with text ad (2 ads shown), against the data when either rich ad or text ad alone was shown (1 ad shown). We did not find any significant improvement in user satisfaction when showing two high quality ads vs. one. In fact, users were slightly less satisfied when shown two ads (rich ad + text ad) compared to when they were shown a rich ad only $(4.05 \pm 0.17$ vs. $4.23 \pm 0.12$ ), presumably because of lower relevance of the text ad, i.e., unlike rich answer-like ad, the text only ad does not expose highly relevant product information, such as product image, model and the price, making users to skip the entire ad block.

\section{GAZE INFERENCE MODELS}

The previous work [22] showed that viewport data may provide a scalable alternative to eye-tracking on mobile devices. We extend the prior work by exploring different probabilistic models to test whether we can improve the accuracy of users' gaze prediction using viewport data. These models are trained and tested on the data described in Section 3.

\subsection{Experimental Setting and Notation}

By gaze inference we refer to assigning a probability of a result being viewed (i.e., gazed), depending on position of the result inside of the viewport and (possibly) visibility of other results. Since the most common layout for results on a mobile phone is linear, i.e., results appear one after another from top to bottom, we focus on the modeling distribution of gaze along the vertical position $y$ in the viewport. For convenience, we re-scale gaze position $y$ from $[0, H]$ to $[0,1]$ dividing it by viewport height $H$. This way, our approach could be generalized to mobile devices with displays of varying size (assuming that display size does not significantly affect gaze). Similarly to [16] we align gaze and viewport data using nearest neighbor interpolation, so that for any given viewport we have a single gaze position and the associated result that was viewed. Since temporal resolution of our viewport data is relatively high (200ms) such approach should be suitable for most practical applications.

\subsection{Uniform Density}

Previous work [22] explored a simple way to share viewport time among the results in the viewport, by assuming that gaze position is uniformly distributed across the entire viewport. According to this approach, the gaze time on a result is proportional to the result's coverage:

$$
\operatorname{coverage}(R, V)=\frac{\operatorname{Area}(R \cap V)}{\operatorname{Area}(V)}
$$

where $R$ and $V$ represent result and viewport bounding boxes and $\operatorname{Area}(X)$ evaluates area of the rectangle. In a setting similar to ours, [22] found that result coverage multiplied by viewport time is well correlated with gaze time.

\subsection{Truncated Normal Density}

Simplistic assumption of gaze being distributed uniformly across the screen might not hold in practice, thus, as an alternative to uniform, we aim to model vertical position of gaze with a normal distribution $N(y ; \mu, \sigma)$ with two parameters $\mu$ and $\sigma$. More precisely, since viewport screen has limited dimensions, using ordinary normal distribution would lead to a mis-specified model, since such choice would assign non-zero probabilities to gaze positions that go beyond the viewport boundaries. To this end, we adopt truncated normal distribution $T N(y ; \mu, \sigma, a, b)$ which assigns zero probability to gaze position outside of the viewport.

Since there is no closed form solution for estimation of $\mu$ and $\sigma$ one needs to estimate their values numerically. In this work we use standard numerical technique available in [18]. In order to perform inference on the amount of time user spent viewing a particular result, one needs to calculate cumulative density function, in this case given by

$$
F_{T N}(y ; \mu, \sigma, a, b)=\frac{\Phi(y)-\Phi(a)}{\Phi(b)-\Phi(a)}
$$

where $\Phi(x)$ is the cumulative density function of the standard normal distribution. To compute the time user spent viewing a particular result we calculate the difference between $F_{T N}(y)$ at top and bottom vertical coordinates of the result in viewport, i.e., the inferred gaze time is proportional to $\left(F_{T N}(\right.$ bottom $\left.)-F_{T N}(t o p)\right)$.

\subsection{Non-Parametric Density}

To capture a more complex distribution shape, we considered a non-parametric approach using kernel density estimation. In this case probability density function of gaze vertical position takes the following form:

$$
f(y)=\frac{1}{N \cdot h} \sum_{j=1}^{N} K\left(y-y_{j}\right)
$$

where $N$ is number of training data points, $K$ is a kernel function with bandwidth $h$ and $y_{j}$ is vertical position of the gaze in the $j$-th viewport. Once $f(y)$ is estimated based on training data $y_{j}$ we can apply it to calculate probability of user looking at an arbitrary vertical position $y$ in the viewport. In this paper, we use the implementation of kernel density estimation available in [18] that uses Gaussian kernel and automatically selects appropriate bandwidth parameter $h$. In order to calculate the probability that user viewed a particular result in the viewport, one needs to integrate $f(y)$ on the interval occupied by the result vertically. In our experiments reported in Section 5.7 we perform this integration numerically using quadrature method with step size $b=1 / 1000$ of the viewport height.

\subsection{Linear Model}

Since our ultimate goal is to infer which exact result did the user look at, static gaze density approaches might be sub-optimal. We argue that one should not need very nuanced, real valued density function to infer which result user is currently viewing, since the set of possibilities is discrete and limited to the few results visible on the phone screen at any given time. Thus, we propose to model allocation of user attention as a discrete distribution conditional on all 
results visible in the viewport:

$$
P\left(G=i \mid R_{1}, \ldots, R_{n_{j}}\right)=\frac{\exp \left(\mathbf{w} \cdot \mathbf{x}_{\mathbf{i}}\right)}{\sum_{i=1}^{n_{j}} \exp \left(\mathbf{w} \cdot \mathbf{x}_{\mathbf{i}}\right)}
$$

where $G$ is a discrete random variable representing index of a viewed result, $i$ is a result index, $R_{1}$ through $R_{n_{j}}$ indices of results visible in $j$-th viewport (there are $n_{j}$ of them). $\mathbf{x}_{\mathbf{i}}$ is a feature vector representing attractiveness of the $i$-th result and $\mathbf{w}$ vector of feature weights. We perform maximum likelihood estimation of model parameters $\mathbf{w}$, by optimizing log-likelihood of training data that is given by

$$
\ell(\mathbf{w})=\sum_{q=1}^{|Q|} \sum_{j=1}^{\left|V_{q}\right|} \log P\left(G=i_{q, j} \mid R_{q, j, 1}, \ldots, R_{q, j, n_{j}}\right)+\lambda\|\mathbf{w}\|^{2}
$$

where $|Q|$ is total number of queries in training data, $q$ is query index, $\left|V_{q}\right|$ is number of viewports for $q$-th query, $j$ is viewport index for query $q, n_{j}$ is number of visible results in $j$-th viewport, $i_{q, j}$ is the index of result viewed in $j$-th viewport (i.e., gaze ground truth) and $\lambda$ is a regularization parameter preventing the model from over fitting the data. The $\log$-likelihood $\ell(\mathbf{w})$ function is a convex function of $\mathbf{w}$ and therefore can be efficiently optimized using gradient based methods. In order to do this, one needs to calculate gradient:

$\nabla \ell(\mathbf{w})=\sum_{q=1}^{|Q|} \sum_{j=1}^{\left|V_{q}\right|}\left(\mathbf{x}_{\mathbf{i}_{\mathbf{q}, \mathbf{j}}}-\sum_{i=1}^{n_{j}} \mathbf{x}_{\mathbf{i}} \times P\left(G=i \mid R_{q, j, 1}, \ldots, R_{q, j, n_{j}}\right)\right)$

We use BFGS[18] iterative method to perform the optimization and train the model.

Features: The above framework offers us a lot of flexibility in terms of using various features to represent relative attractiveness of each result in the viewport. We experiment with the following features. Coverage is defined in Equation 1. Exposure is very similar to coverage, except that it measures the fraction of the result visible to the user. Exposure can be calculated by swapping arguments in Equation 1, i.e., Coverage $(V, R)$. Top is defined as the top-most vertical coordinate of a result relative to the viewport's top. To capture more complex relationship between result position in the viewport and probability being viewed, we include a family of five features representing overlap between the result and five disjoint screen areas (these areas partition the viewport's vertical space equally). We denote these features as ScreenAreas5. The overlap between the result $R$ and ScreenArea $_{k}$ can be calculated as Coverage $\left(\right.$ ScreenArea $\left._{k}, R\right)$. They allow us to comibine result position information, similar to gaze density approaches, with other attractiveness features of a result. One could argue that gaze distribution might change depending on whether user scrolls the page or not. To this end, we include two sets of screen area features to capture potentially different gaze distribution depending on whether user scrolled vertically or not. We compute Cartesian product of ScreenAreas5 and binary indicator InitialViewport. InitialViewport evaluates to true if top of the viewport lies in $[0, H / 2]$, where $H$ is the height of the phone's screen (the same as viewport height if no zoom performed).

\subsection{Evaluation Metrics}

To evaluate the quality of the various gaze prediction models we use Pearson's correlation coefficient. To ensure that

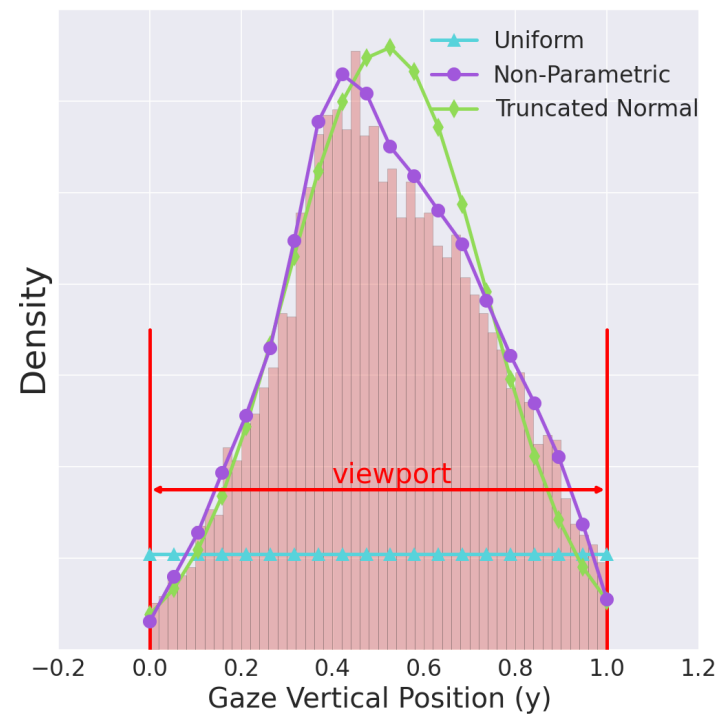

Figure 8: Static densities of gaze along the vertical dimension of the phone's viewport (normalized from $[0,1]$ with 0 being the top of the viewport, and 1 being the bottom.

the model performance generalizes to out of sample data and that we do not over-report the accuracy, we perform leave one-user-out cross validation, so that data used in training stage does not come from the same participant as the data we use for model evaluation. A good model should accurately predict the time user spent viewing a particular result in a given query event, therefore we use time on (query, result) as a single data point in calculating the correlation. We adopt similar evaluation methodology as prior work [22], and instead of absolute value of time on (query, result) correlate fraction of time on result, defined as time in result divided on time user spent on the entire query.

\subsection{Results}

Figure 8 shows the histogram of user gaze positions along the vertical dimension of the viewport. Also, the Figure shows density functions for uniform distribution, truncated normal $(\mu=0.515, \sigma=0.216)$ and non-parametric densities that were fitted on participants' gaze data. Overall, the truncated normal distribution comes close to the empirical gaze histogram. However, as seen in the figure, the nonparametric distribution provides an even better fit, closely following the small variations in the histogram. We now turn to quantitative evaluation of the described approaches. Table 2 summarizes performance of different approaches on four subsets of the data: rich ad, text ad, web and all result types. In each cell of the table, we report Pearson's correlation between the ground truth (gaze time) and the model's prediction on inferred gaze time. The prediction is averaged across several folds of cross validation. Surprisingly, the simplest among gaze density approaches - Uniform distribution - outperforms Truncated Normal and even more nuanced Non-Parametric approaches by a significant margin. We believe this is due to the inability of static densities 


\begin{tabular}{|c|c|c|c|c|c|}
\hline Model & Features & $\begin{array}{l}\text { Rich Ad } \\
(\mathrm{N}=640)\end{array}$ & $\begin{array}{l}\text { TextAd } \\
(\mathrm{N}=608)\end{array}$ & $\begin{array}{c}\text { Web } \\
(\mathrm{N}=5271)\end{array}$ & $\begin{array}{c}\text { All } \\
(\mathrm{N}=6609)\end{array}$ \\
\hline Uniform & $\mathrm{n} / \mathrm{a}$ & $0.676(\mathrm{n} / \mathrm{a})$ & $0.627(\mathrm{n} / \mathrm{a})$ & $0.433(\mathrm{n} / \mathrm{a})$ & $0.513(\mathrm{n} / \mathrm{a})$ \\
\hline Truncated Normal & $\mathrm{n} / \mathrm{a}$ & $.660(-2 \%)$ & $0.511(-18 \%)$ & $0.344(-20 \%)$ & $0.466(-9 \%)$ \\
\hline Non-Parametric & $\mathrm{n} / \mathrm{a}$ & $0.656(-2 \%)$ & $0.544(-13 \%)$ & $0.356(-17 \%)$ & $0.496(-3 \%)$ \\
\hline M0 & Coverage & $0.662(-2 \%)$ & $0.582(-7 \%)$ & $0.445(+2 \%)$ & $0.544(+6 \%)$ \\
\hline M1 & Coverage, Top & $0.675(0 \%)$ & $0.635(+1 \%)$ & $0.492(+13 \%)$ & $0.616(+20 \%)$ \\
\hline M2 & $\begin{array}{l}\text { Coverage, Top, } \\
\text { Exposure }\end{array}$ & $0.691(+2 \%)$ & $0.646(+3 \%)$ & $0.510(+17 \%)$ & $0.633(+23 \%)$ \\
\hline M3 & $\begin{array}{c}\text { Coverage, } \\
\text { Exposure, } \\
\text { ScreenAreas5 }\end{array}$ & $0.701(+3 \%)$ & $0.633(+0 \%)$ & $0.536(+23 \%)$ & $0.650(+26 \%)$ \\
\hline M4 & $\begin{array}{c}\text { Coverage, } \\
\text { Exposure, } \\
\text { ScreenAreas } 5 \times \\
\text { InitialViewport }\end{array}$ & $\begin{array}{c}0.705 \\
(+4 \%)\end{array}$ & $\begin{array}{c}0.649 \\
(+3 \%)\end{array}$ & $\begin{array}{c}0.558^{*} \\
(+28 \%)\end{array}$ & $\begin{array}{c}0.671^{*} \\
(+30 \%)\end{array}$ \\
\hline
\end{tabular}

Table 2: Comparison of gaze prediction models based on Pearson's correlation between ground truth on gaze time per result and model predictions (inferred from viewport data). * indicates significant difference compared to Uniform approach with p-value $<0.001$.

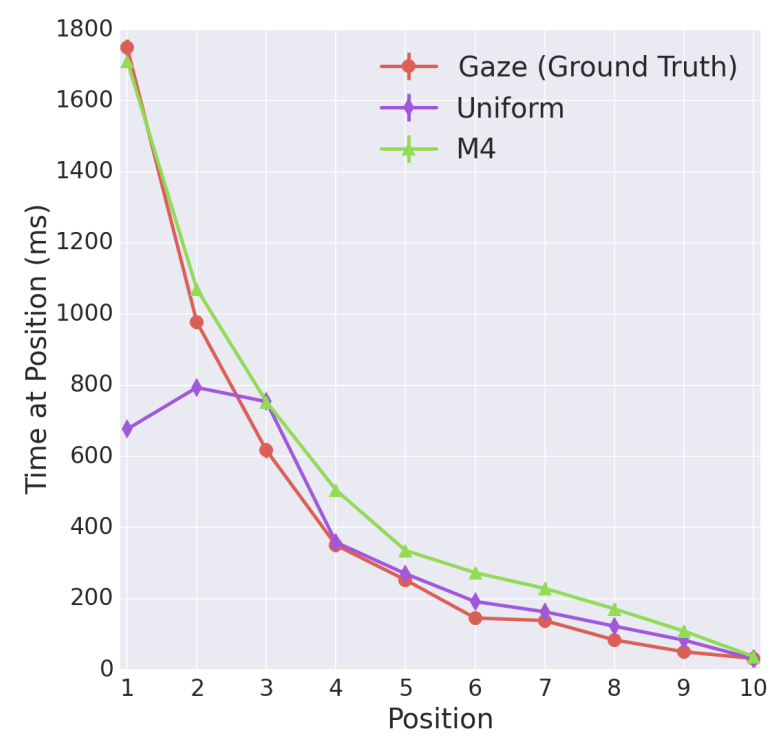

Figure 9: View time on each result position calculated from users' gaze (ground truth), and compared to the predictions of the uniform and M4 models.

to capture complex behavior that can change depending on what is present in the viewport. On the other hand, even a relatively simple linear model Mo, that uses the same information as the Uniform baseline, is able perform slightly better than Uniform distribution. Including Top into the model M1 outperforms Uniform baseline by $20 \%$ and improves Pearson's correlation from 0.513 to 0.616 on all result types. More complex versions of the linear model M2-M4 are able to further improve the correlation to 0.671 which is $30 \%$ better than the baseline model. To understand our results further we examined the ability of our models to predict the variation in gaze time along result position. Figure
9 shows users' gaze data (in red), the prediction of the uniform model (in purple), and M4 version of the linear model (in green). Consistent with prior work [22], we find similar behavior exhibited by the uniform model that assigns higher view time to position 2-3 than to the first result position. Interestingly, our linear model $\mathbf{M} 4$ is able to correct this oddity and demonstrate a more understandable pattern of decay of view time with position, which is more similar to observed gaze data. Together, Table 2 and Figure 9 show that our model is not only able to predict viewed results more accurately than previous work, but also exhibits more sensible behavior in qualitative results.

\section{DISCUSSION}

We summarize the main contributions of this work. This is the first study to analyze user gaze behavior and search satisfaction in presence of rich, answer-like ad format on mobile phones. Second, we showed that rich ad formats draw more attention than traditional text ads or organic results at the same position, and result in higher searcher satisfaction compared to showing no ads. Third, we studied how user gaze varies along the vertical dimension (as the user scrolls down the SERP), as well as the horizontal dimension within the rich ad. Fourth, we developed various gaze inference models that improve accuracy of attention measurement for $30 \%$ on both organic and sponsored results.

\section{Effect of position and ad format on gaze}

To our knowledge, this is the first study to investigate user gaze behavior on search elements that can be swiped horizontally. The answer-like ads used in our study were all swipable - the initial screen showed 2 full offers and $50 \%$ of a third offer, and users could swipe to see more offers ( 5 offers in total, and more available upon click).

In the absence of a swipe, we found that attention strongly decays from the leftmost position to the right. This is consistent with previous findings from desktop where gaze density is known to be shaped like a golden triangle [20, 23], with a focus on the top-left that decays as we move towards the right or bottom on the SERP. Somewhat surprisingly, in the presence of a swipe, we found that the attention preference 
for the leftmost position disappears and all positions seem to be equally viewed. This fact could be used to design appropriate user models of swipable UIs, e.g., consider all the items appearing to the left of the rightmost item visible in the viewport as viewed by the user. Number of offers in the swipable unit may also have an effect, e.g., for units with large number of sub-results we may find differential viewing with more time spent on some offers compared to others.

As expected, and similar to desktop settings, on mobile phones, we found that attention on organic results (when no ads are shown) decays with position as we go down the search results page. We found that on mobile phones, the text ad gets less attention than an organic web result at the same position, which is likely to ads blindness (e.g., [4]).

In contrast to the above, a rich format ad gets more attention than an organic result at the same position. In fact, we found that rich format ad gets significantly more attention than a text ad at the same position. This could be due to multiple reasons, such as the higher visual prominence of the rich ad format (e.g., it contains image thumbnails), its interactive nature (users can swipe to see more ad offers) or higher perceived relevance, i.e., the rich ad format previews the facets that users care about: price, product picture and the store where it's available.

\section{Inferring gaze from viewport}

Due to the relatively high cost of existing commercial eye trackers and time-consuming process of running in-lab user studies, in-lab user studies are usually limited to 20-50 users. We explored whether viewport data could be used at scale to reliably infer user gaze on a per-element basis on the SERP. We developed various models and compared their performance on gaze prediction to previous state-of-the-art approaches. In particular, we explored different ways to weight each pixel within the viewport to better infer the gaze time on each result, such as weighting based on a uniform distribution, truncated normal and non-parametric gaze density. In addition, we explored using feature based linear models that predict which element the user looked at based on combination of features described in Section 5.5. Our results demonstrated that feature based models are more accurate and improve Pearson's correlation between gaze and model predictions on $30 \%$ (from 0.513 to 0.671 ). Unlike previous methods [22], our approach does not suffer from over-counting viewport time on second and third result positions, and aligns with observed gaze data more closely (as shown in Figure 9).

One benefit of using a simple linear models is that it is relatively easy to interpret the coefficients, hence the model's predictions. Model M4 assigns positive weights to both coverage and exposure features: $\mathrm{w}$ (Coverage) $=1.03$ and $\mathrm{w}($ Exposure $)=1.31$. Figure 10 shows the weights for ScreenAreas 5 features sliced by whether it belongs to the InitialViewport or not. Each curve on the figure has five data points corresponding to the five screen areas defined in Section 5.5. Interestingly, we see that the model assigns very different weights to each screen area, depending on the viewport. That is, when viewport remains on top of the page and InitialViewport = True, $\mathbf{M} 4$ assigns higher view probabilities to results positioned higher in the phone screen. However, when the user scrolls down, the model assigns higher probabilities to the central part of the screen. Note that the feature weight on the top area of the screen in the initial

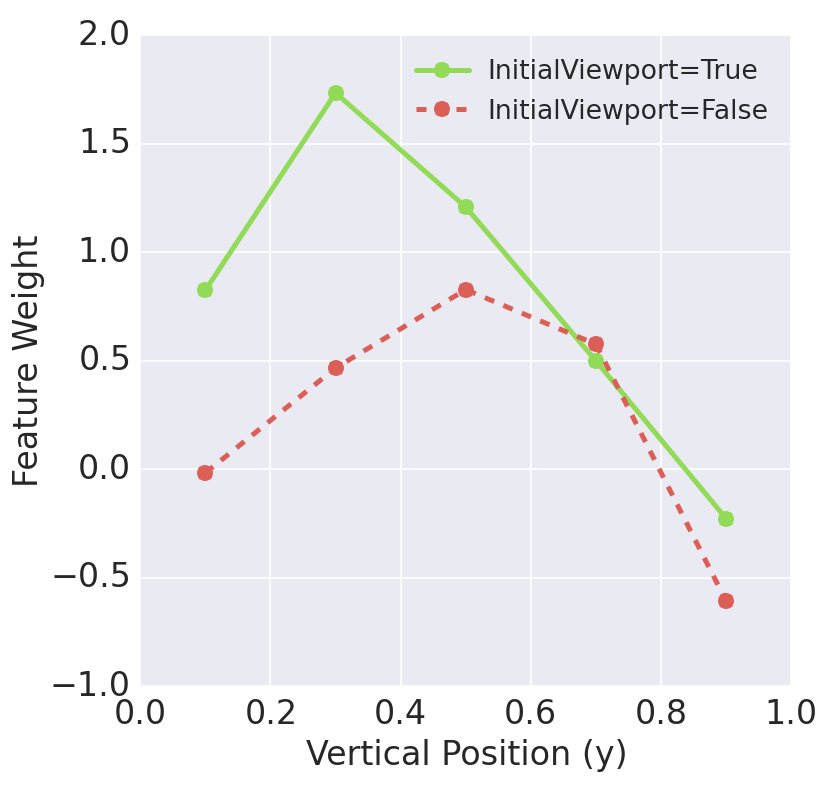

Figure 10: Weights of the ScreenAreas5 features in model M4.

viewport is substantially smaller than the weight of the adjacent area due to the presence of the search box that usually occupies the topmost part of the page (see Figure 1), which in our study received less gaze time as we predefined the queries for the user tasks.

\section{User satisfaction}

For commercial queries, we found that showing a high quality ad does not always translate to improved user satisfaction. While showing answer-like ads significantly increased user satisfaction on the SERP compared to not showing any ads, this was not the case for showing regular text ads. It is possible that providing answer-like information on various options about the product, including image, pricing and merchant makes the rich format ad more valuable to the user, especially for comparative shopping tasks, thereby resulting in higher user satisfaction. It's also valuable for "price check" intents, since rich ad format provides user with a range of prices for the same product. To summarize, we found that the rich ad drew longer gaze time on ad relative to other elements on SERP and was associated with higher user satisfaction.

\section{Limitations}

Since commercial eye-tracking is not yet available at scale, we were limited to conducting this study on a small set of 24 users in lab settings. We acknowledge that the relatively small dataset in this study may limit its generalizability to the real user population as a whole. This is an unfortunate limitation of virtually any eye study [24, 13, 8, 14, 22, 20, 23]. On the positive side, we were able to collect a rich dataset on user behavior in the lab by simultaneously recording user's gaze, their scrolling behavior, and the visible content on their phone. Also, to enable better generalization of our study's findings to the large user population, we randomly selected our users to belong to different gender, race and ages to increase the diversity. 
Given the small number of users and limited time per user, our study design was limited to a few experimental conditions that either showed one rich format ad, or a traditional text ad, or both, or no ad. In future, we would like to explore additional variations within rich ad formats (e.g., the effect of image size, amount of information, star ratings, reviews).

The current models attempt to infer user gaze from viewport data such as coverage, exposure, and position of elements on the phone screen. Other studies [21, 7] have shown that the actual content and presentation can also affect user behavior. In our future work we plan to incorporate more complex features and experiment with non-linear models.

\section{CONCLUSIONS}

In this paper, we studied how users' eye gaze and satisfaction are impacted by the presence of answer-like ads and their formats. In particular, we compared the effect of showing a answer-like ad that can be swiped horizontally vs. a regular text ad vs. both vs. no ads. We found that showing a rich ad attracted more attention on the ad unit compared to a regular text ad or organic result at the same position, and better satisfied users compared to showing no ads. We extended previous work by developing gaze inference models that provide improve accuracy of attention measurement on a per-result basis. Together, this shows that viewport data can be extremely valuable for studies of user behavior in search and ads, due to its scalability and accuracy in measuring user attention on mobile devices.

\section{REFERENCES}

[1] Retrieved from http://www.eyetracking-glasses.com/fileadmin/user_ upload/documents/smi_etg2w_flyer_naturalgaze.pdf, 2015.

[2] Retrieved from https://en.wikipedia.org/wiki/3D_ projection\#Perspective_projection, 2016.

[3] R. Biedert, A. Dengel, G. Buscher, and A. Vartan. Reading and estimating gaze on smart phones. In Proc. of the Symposium on Eye Tracking Research and Applications, pages 385-388. ACM, 2012.

[4] G. Buscher, S. T. Dumais, and E. Cutrell. The good, the bad, and the random: an eye-tracking study of ad quality in web search. In Proc. of SIGIR, pages 42-49. ACM, 2010.

[5] M. C. Chen, J. R. Anderson, and M. H. Sohn. What can a mouse cursor tell us more?: correlation of eye/mouse movements on web browsing. In Proc. of SIGCHI Extended Abstracts, pages 281-282, 2001.

[6] E. Cutrell and Z. Guan. What are you looking for?: an eye-tracking study of information usage in web search. In Proc. of SIGCHI, pages 407-416. ACM, 2007.

[7] F. Diaz, R. White, G. Buscher, and D. Liebling. Robust models of mouse movement on dynamic web search results pages. In Proc. of CIKM, pages 1451-1460. ACM, 2013.

[8] C. Eickhoff, S. Dungs, and V. Tran. An eye-tracking study of query reformulation. In Proc. of SIGIR, pages 13-22. ACM, 2015.

[9] L. A. Granka, T. Joachims, and G. Gay. Eye-tracking analysis of user behavior in WWW search. In Proc. of SIGIR, pages 478-479. ACM, 2004.
[10] Z. Guan and E. Cutrell. An eye tracking study of the effect of target rank on web search. In Proc. of SIGCHI, pages 417-420. ACM, 2007.

[11] Q. Guo and E. Agichtein. Towards predicting web searcher gaze position from mouse movements. In Proc. of SIGCHI Extended Abstracts, pages 3601-3606, 2010.

[12] Q. Guo, H. Jin, D. Lagun, S. Yuan, and E. Agichtein. Mining touch interaction data on mobile devices to predict web search result relevance. In Proc. of SIGIR, pages 153-162. ACM, 2013.

[13] Q. Guo, D. Lagun, and E. Agichtein. Predicting web search success with fine-grained interaction data. In Proc. of CIKM, pages 2050-2054. ACM, 2012.

[14] K. Hofmann, B. Mitra, F. Radlinski, and M. Shokouhi. An eye-tracking study of user interactions with query auto completion. In Proc. of CIKM, pages 549-558. ACM, 2014.

[15] J. Huang and A. Diriye. Web user interaction mining from touch-enabled mobile devices. Proc. of HCIR, 2012.

[16] J. Huang, R. White, and G. Buscher. User see, user point: gaze and cursor alignment in web search. In Proc. of SIGCHI, pages 1341-1350. ACM, 2012.

[17] J. Huang, R. W. White, and S. Dumais. No clicks, no problem: using cursor movements to understand and improve search. In Proc. of SIGCHI, pages 1225-1234. ACM, 2011.

[18] E. Jones, T. Oliphant, P. Peterson, et al. SciPy: Open source scientific tools for Python, 2001-. [Online; accessed 2016-01-19].

[19] D. Lagun and E. Agichtein. Viewser: enabling large-scale remote user studies of web search examination and interaction. In Proc. of SIGIR, pages 365-374. ACM, 2011.

[20] D. Lagun and E. Agichtein. Effects of task and domain on searcher attention. In Proc. of SIGIR, pages 1087-1090. ACM, 2014.

[21] D. Lagun and E. Agichtein. Inferring searcher attention by jointly modeling user interactions and content salience. In Proc. of SIGIR, pages 483-492. ACM, 2015.

[22] D. Lagun, C.-H. Hsieh, D. Webster, and V. Navalpakkam. Towards better measurement of attention and satisfaction in mobile search. In Proc. of SIGIR, pages 113-122. ACM, 2014.

[23] Z. Liu, Y. Liu, K. Zhou, M. Zhang, and S. Ma. Influence of vertical result in web search examination. In Proc. of SIGIR, 2015.

[24] L. Lorigo, M. Haridasan, H. Brynjarsdóttir, L. Xia, T. Joachims, G. Gay, L. Granka, F. Pellacini, and B. Pan. Eye tracking and online search: Lessons learned and challenges ahead. JASIST, 59(7):1041-1052, 2008.

[25] V. Navalpakkam, L. Jentzsch, R. Sayres, S. Ravi, A. Ahmed, and A. Smola. Measurement and modeling of eye-mouse behavior in the presence of nonlinear page layouts. In Proc. of $W W W, 2013$.

[26] K. Rodden, X. Fu, A. Aula, and I. Spiro. Eye-mouse coordination patterns on web search results pages. In Proc. of SIGCHI Extended Abstracts, 2008. 